\title{
Ultrafast broadband optical spectroscopy for quantifying subpicometric coherent atomic displacements in $\mathrm{WTe}_{2}$
}

\author{
Davide Soranzio $\odot,{ }^{1}$ Maria Peressi $\odot,{ }^{1}$ Robert J. Cava, ${ }^{2}$ Fulvio Parmigiani $\odot,{ }^{1,3,4}$ and Federico Cilento $\odot^{4, *}$ \\ ${ }^{1}$ Dipartimento di Fisica, Università degli Studi di Trieste, 34127 Trieste, Italy \\ ${ }^{2}$ Department of Chemistry, Princeton University, Princeton, New Jersey 08544, USA \\ ${ }^{3}$ International Faculty, University of Cologne, Albertus-Magnus-Platz, 50923 Cologne, Germany \\ ${ }^{4}$ Elettra-Sincrotrone Trieste S. C. p. A., Strada Statale 14, km 163.5, 34149 Basovizza, Trieste, Italy
}

(Received 22 May 2019; published 5 December 2019)

\begin{abstract}
Here we show how time-resolved broadband optical spectroscopy can be used to quantify, with femtometer resolution, the oscillation amplitudes of coherent phonons through a displacive model without free tuning parameters, except an overall scaling factor determined by comparison between experimental data and density functional theory calculations. WTe ${ }_{2}$ is used to benchmark this approach. In this semimetal, the response is anisotropic and provides the spectral fingerprints of two $A_{1}$ optical phonons at $\approx 8$ and $\approx 80 \mathrm{~cm}^{-1}$. In principle, this methodology can be extended to any material in which an ultrafast excitation triggers coherent lattice modes modulating the high-energy optical properties.
\end{abstract}

DOI: 10.1103/PhysRevResearch.1.032033

Several fundamental properties of materials, including the electrical and thermal conductivities, are influenced by lattice vibrations [1]. Recently, the possibility to control such behaviors by resonant coupling of ultrashort light pulses to specific lattice modes has been proved [2,3]. Conversely, coherent phonon spectroscopy has emerged as a powerful method to directly observe, in the time domain, coherent lattice vibrations [4-6]. Despite the many studies, coherent phonons have been exploited primarily to characterize ground-state properties [7-10], rather than controlling the material properties. This observation motivates the quest to learn how specific lattice vibrations affect the electronic behavior in the vicinity of the Fermi energy. Structural dynamics experiments, such as timeresolved x-ray diffraction [11] and time-resolved electron diffraction [12], are currently used to measure the amplitude of synchronized collective excitations of the atoms in a solid, i.e., coherent phonon modes. Yet, subpicometer displacements in complex materials are very challenging to resolve.

In time-resolved reflectivity experiments, such modes appear as ultrafast oscillations of the probe signal [13,14]. Their amplitude has been related to the atomic shifts for singleelement materials $[15,16]$ by using single-frequency measurements. In materials with complex unit cells, a larger number of phonon modes is present and a method that avoids correlations in the estimation of individual amplitudes is required. Here, we report on an approach to estimate the nonequilibrium atomic displacements of coherent optical phonon modes by

\footnotetext{
*federico.cilento@elettra.eu

Published by the American Physical Society under the terms of the Creative Commons Attribution 4.0 International license. Further distribution of this work must maintain attribution to the author(s) and the published article's title, journal citation, and DOI.
}

detecting the modulation induced in a broadband reflectivity probe signal, which is central to obtain reliable signatures of the modes. Density functional theory (DFT) calculations are applied to a displacive model to simulate the time-resolved optical reflectivity signals and to estimate the coherent phonon amplitudes in the tens of femtometers regime.

At present, our method is applied to the orthorhombic semimetallic (Td) phase of tungsten ditelluride $\left(\mathrm{WTe}_{2}\right)$, a transition metal dichalcogenide that has recently gained interest for showing unusually high and nonsaturating magnetoresistance [17] along with a possible type-II Weyl semimetal character [18]. $\mathrm{WTe}_{2}$ has a layered structure belonging to the space group Pmn2 ${ }_{1}$ [19] (see Fig. S1 in the Supplemental Material [20]) with in-plane covalent bonding and mainly van der Waals interactions holding together the individual layers. Furthermore, it has been shown that its electronic, optical, and topological properties are influenced by strain forces [18,21], which can be induced through nonequilibrium perturbations [22], allowing an ultrafast control of the functionalities of this material.

Expressly, we focus on two $\mathrm{A}_{1}$ coherent optical phonon modes at $\approx 8$ and $\approx 80 \mathrm{~cm}^{-1}$. They are composed of nonequilibrium displacements of the atoms along the $\mathbf{y}$ and $\mathbf{z}$ crystallographic directions. The $\approx 8 \mathrm{~cm}^{-1}$ optical phonon is a uniform in-plane shift of the atoms, estimated to be $\approx 350 \mathrm{fm}$ using a $\approx 230 \mu \mathrm{J} / \mathrm{cm}^{2}$ absorbed pump fluence, while the $\approx 80 \mathrm{~cm}^{-1}$ mode corresponds to atomic displacements of few tens of femtometers which depend on the specific atom. A more detailed description will be given further on in this work.

High-quality tungsten ditelluride samples were grown as reported in Ref. [17]. The presence of defects was previously studied [17,23] and has negligible impact on our estimate of the average displacements. In order to identify the in-plane crystallographic axes, low energy electron diffraction (LEED) images were acquired under ultrahigh vacuum conditions. 
(a)

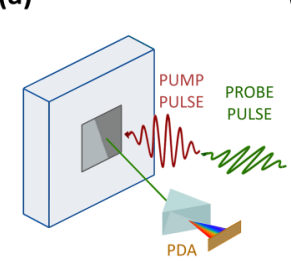

(b)

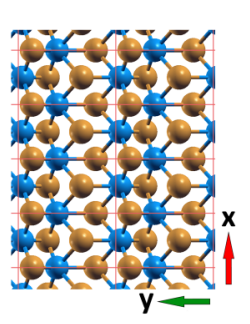

(c) 2.

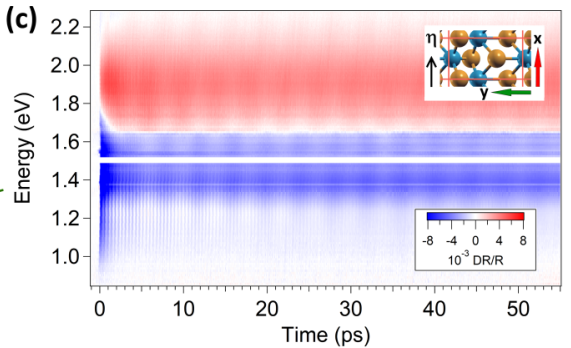

(d)

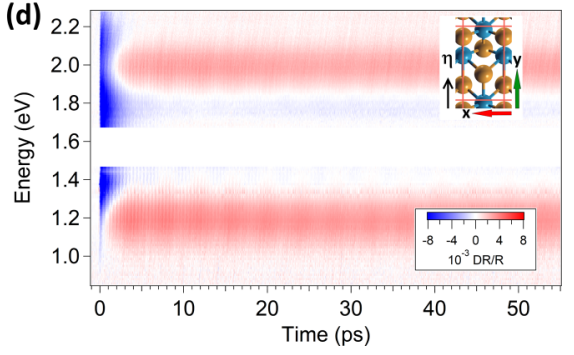

FIG. 1. (a) Sketch of the time-resolved broadband reflectivity experiment; the reflected probe beam is dispersed through a glass prism and detected by a photodiode array (PDA) detector. (b) Projection of a $\mathrm{WTe}_{2}$ layer on the $\boldsymbol{x y}$ plane with blue tungsten and orange tellurium atoms showing multiple unit cells delimited by red lines; other projections are reported in Ref. [20]. [(c), (d)] Ultrafast broadband $\mathrm{DR} / \mathrm{R}$ images for $\mathrm{WTe}_{2}$ with the probe beam polarization along (c) $\mathbf{x}$ and (d) $\mathbf{y}$, both taken at $\approx 710 \mu \mathrm{J} / \mathrm{cm}^{2}$ absorbed pump fluence and $1.55-\mathrm{eV}$ pump photon energy at $T=295 \mathrm{~K}$.

Time-resolved reflectivity experiments [sketch of the setup in Fig. 1(a)] were performed using a Ti:sapphire femtosecond (fs) laser system, delivering, at a repetition rate of $250 \mathrm{kHz}$, $\approx 50 \mathrm{fs}$ light pulses at a wavelength of $800 \mathrm{~nm}(1.55 \mathrm{eV})$. The broadband $(0.8-2.3 \mathrm{eV})$ supercontinuum probe beam was generated using a sapphire window.

DFT simulations were carried out using norm-conserving (NC) [24] scalar relativistic [25] pseudopotentials with the generalized gradient approximation (GGA) in the PerdewBurke-Ernzerhof (PBE) parametrization for the exchangecorrelation functional [26] chosen from the PseudoDojo database [27,28]. Structural optimizations and zone-center optical phonon calculations in the framework of density functional perturbation theory (DFPT) were performed using the QUANTUM ESPRESSO (QE) [29] suite of codes. We calculated the diagonal macroscopic dielectric tensor components through the YAMBO code [30] at the independent-particle (IP) level, starting from the calculated wave functions and eigenvalues obtained through QE [20].

The normalized time-resolved differential reflectivity (DR/R), measured in a near-normal incidence geometry, revealed a large anisotropy consistent with the twofold in-plane symmetry of $\mathrm{WTe}_{2}$ [Fig. 1(b)] also reported for equilibrium electrodynamics [31].

In Figs. 1(c) and 1(d), the broadband probe beam polarization was set parallel to the $\mathbf{x}(\eta \| \mathbf{x})$ and $\mathbf{y}(\eta \| \mathbf{y})$ crystallographic axes respectively with pump beam polarization kept perpendicular to the probe one. Measurements were acquired with a $\approx 710 \mu \mathrm{J} / \mathrm{cm}^{2}$ absorbed pump fluence and $1.55-\mathrm{eV}$ pump photon energy at $T=295 \mathrm{~K}$. A region of the spectrum

was disturbed by the scattering of pump beam photons from the sample, so it was removed.

The temporal evolution of the reflectivity is directly related to the electronic and ionic degrees of freedom $[4,13,14]$. In general, the DR/R is a superposition of signals due to transient variations of electronic density of states and population. We describe the temporal evolution after the perturbation (time zero, $t=0$ ) as

$$
\begin{aligned}
\frac{D R}{R}(t, h v)= & G(t)\left[\sum_{i} A_{i}(h v) e^{-t / \tau_{R i}}+B(h v) e^{-t / \tau_{\text {heat }}}\right. \\
& \left.+\sum_{j} C_{j}(h v) \cos \left(\omega_{j} t+\phi_{j}\right) e^{-t / \tau_{p_{j}}}\right],
\end{aligned}
$$

where $G(t)$ represents the pump and probe pulses' cross correlation and $A_{i}(h v), B(h v)$, and $C_{j}(h v)$ denote the amplitudes of three different phenomena: (i) electronic relaxation phenomena with time constants $\tau_{R i}$, (ii) heating contribution with a characteristic time $\tau_{\text {heat }}$, and (iii) oscillations due to coherent phonons with angular frequency $\omega_{j}$, initial phase $\phi_{j}$, and decay time $\tau_{P j}$.

After the pump pulse excitation, electron-electron and electron-phonon scattering processes constitute the main incoherent relaxation phenomena in the first hundreds of femtoseconds and are responsible for the initial exponential decay of the DR/R signal. After a few picoseconds, the differential reflectivity $\mathrm{DR} / \mathrm{R}$ reached a plateau, showcasing an offset with respect to the equilibrium value. It vanished in almost $1 \mathrm{~ns}$ [20]. Because of its timescale, this effect is likely to be associated with local lattice heating, as described in Ref. [32] for transition metals. For our purposes, we can consider the offset as a constant over the temporal range explored in Figs. 1(c) and 1(d).

The temporal profiles are very well fitted by using Eq. (1) [20]. Their sign and magnitude vary with the photon energy, while the main relaxation presents a time constant which is almost the same and equal to $\approx 1$ ps with only small differences, as previously found in Ref. [33] at $h v=1.55 \mathrm{eV}$.

Subtracting an exponential fit function from the experimental signals allows the extraction of the coherent component of the DR/R signal. The resulting signal displays marked periodic modulations in the time domain, arising from the excitation of coherent phonon modes. The most prominent oscillating features, as detected by fast Fourier transform (FFT) and in Ref. [33], have frequencies of $7.9 \pm 0.4 \mathrm{~cm}^{-1}$ and $79.7 \pm 0.4 \mathrm{~cm}^{-1}$. For notation purposes, we refer to these modes with the labels $8 \mathrm{~cm}^{-1}$ and $80 \mathrm{~cm}^{-1}$. The associated time-decay constants, derived by fitting the modulations with two exponentially-damped cosine waves, are $\tau_{P 1}=77 \pm 4 \mathrm{ps}$ for the $8 \mathrm{~cm}^{-1}$ mode and $\tau_{P 2}=12.1 \pm 0.2 \mathrm{ps}$ for the $80 \mathrm{~cm}^{-1}$ mode. DFPT results indicate that these frequencies are linked to $A_{1}$ zone-center optical phonon modes, represented in Figs. 2(a) and 2(b). The $8 \mathrm{~cm}^{-1}$ mode can be pictured as adjacent layers moving in antiphase, while the $80 \mathrm{~cm}^{-1}$ involves more complex in-plane and out-of-plane movements. The smaller value of the experimental frequency for the $8 \mathrm{~cm}^{-1}$ mode with respect to the DFPT result $\left(10.30 \mathrm{~cm}^{-1}\right)$ can be 

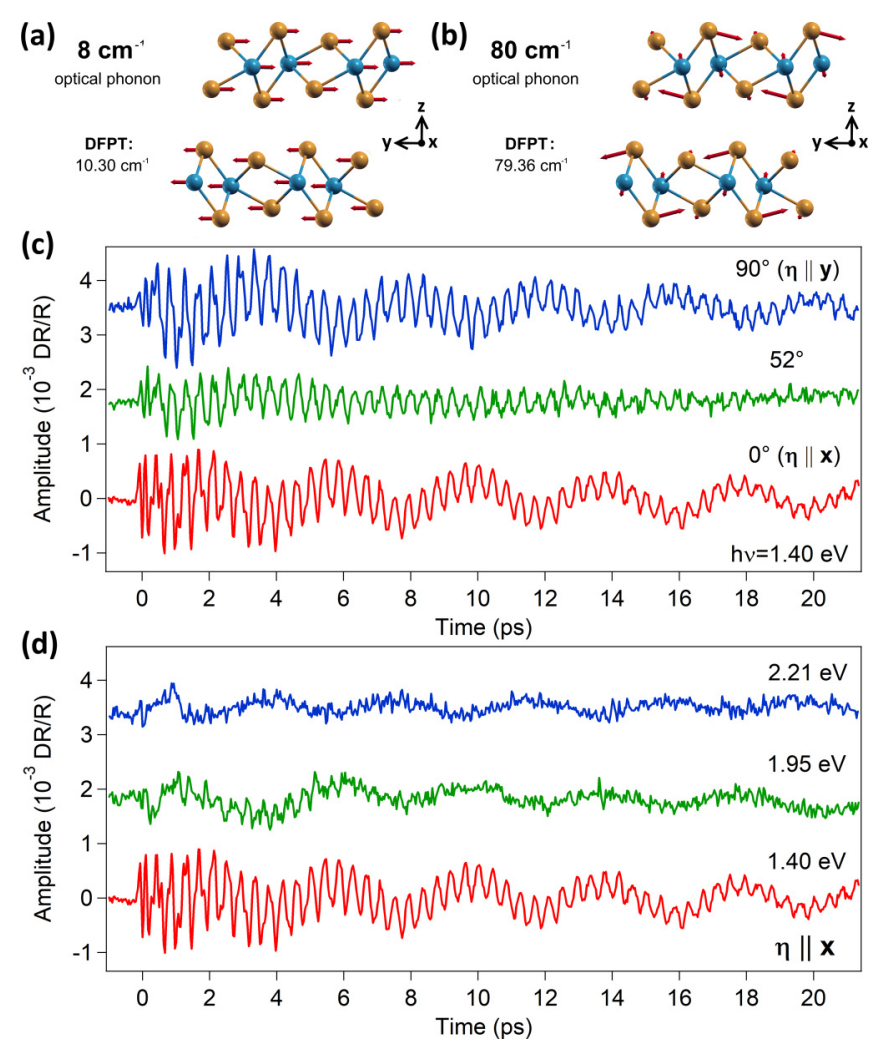

FIG. 2. DFPT eigendisplacements and frequencies for the (a) $8 \mathrm{~cm}^{-1}$ and (b) $80 \mathrm{~cm}^{-1}$ optical modes; the tungsten atoms are blue, whereas the tellurium ones are orange. [(c), (d)] Oscillatory signal due to coherent phonons (the two upper curves are shifted from the central $\mathrm{DR} / \mathrm{R}=0$ ) (c) at $1.40 \mathrm{eV}$ photon energy for different probe beam polarization angles, showing a $\pi$ phase shift for $8 \mathrm{~cm}^{-1}$ mode between the two perpendicular configurations while the $80 \mathrm{~cm}^{-1}$ mode appears to be unaffected, and (d) for $\eta \| \mathbf{x}$ as a function of the photon energy, evidencing a $\pi$ phase shift for the $8 \mathrm{~cm}^{-1}$ mode when comparing the infrared $(1.40 \mathrm{eV})$ to the visible $(2.21 \mathrm{eV})$ spectral regions.

attributed to a red shift as temperature or fluence are increased [34]. The marked difference between the time constants of the two modes could be linked to the different types of perturbation of the interatomic bonds induced by the associated displacements. The $8 \mathrm{~cm}^{-1}$ mode [Fig. 2(a)] only alters the interplanar, mainly van der Waals, interactions, whereas the $80 \mathrm{~cm}^{-1}$ mode [Fig. 2(b)] induces a modification of the inplane covalent bonds. A more detailed DFPT analysis could provide a rigorous basis for this intuitive argument, which is beyond the scope of the present work.

Weaker additional $\mathrm{A}_{1}$ contributions at $116.5 \pm 0.4$, $132.2 \pm 0.4$, and $210.2 \pm 0.4 \mathrm{~cm}^{-1}$ frequency appear as beats in the DR/R signal in the first hundreds of femtoseconds.

When switching the probed direction from $\eta \| \mathbf{x}$ to $\eta \| \mathbf{y}$, a $\pi$ phase change for the $8 \mathrm{~cm}^{-1}$ mode [Fig. 2(c)] was clearly registered for most of the probe photon energies. Analogous $\pi$ phase changes were found by comparing the temporal profiles taken at different probe photon energies using the same polarization for the same mode [Fig. 2(d)]. These phase differences can be explained in terms of the peculiar anisotropy of the dielectric function and were reproduced through our numerical simulations (see the discussion for Fig. 4).
In order to model the effects of the coherent phonons on the reflectivity, we first calculated the wave functions and eigenvalues for the equilibrium configuration using $\mathrm{QE}$ and the macroscopic dielectric tensor diagonal components and the reflectivity curves through YAMBO. Then, we considered four out-of-equilibrium configurations labeled as 0 and $\pi$ phases, corresponding to displacements in opposite directions, for each of the coherent phonon modes, using the eigendisplacements obtained through DFPT with respect to the equilibrium positions. The optical phonons led to modifications of the electronic band structure that, although small, were beyond the numerical accuracy and were confirmed by linearly rescaling the effects of larger perturbations [20]. For each configuration, we calculated the associated reflectivity and DR/R with respect to the equilibrium configuration. These curves describe the effect of the optical phonons on a quasiequilibrium adiabatic system. Indeed, a few picoseconds after time zero, the system relaxed through electron-phonon scattering processes and the incoherent part of the DR/R signal reached a plateau having a decay time much larger than the coherent phonon period and damping timescale.

Experimentally, the effects of each single optical phonon mode on the $\mathrm{DR} / \mathrm{R}$ were isolated with the following procedure. For the $8 \mathrm{~cm}^{-1}$ phonon, we took the mean value over one period of the $80 \mathrm{~cm}^{-1}$ modulation around two consecutive $8 \mathrm{~cm}^{-1}$ extrema (Fig. 3). For the $80 \mathrm{~cm}^{-1}$ oscillation, we directly used the spectral profiles at the extrema, considering that the period of the slower phonon is about ten times larger. The spectral profiles were collected after the main relaxation peak, as precisely indicated in the insets. The difference between the spectral profiles at the maximum ( 0 phase) and minimum ( $\pi$ phase) of the coherent phonons, named with respect to the $\eta \| \mathbf{x} \mathrm{DR} / \mathrm{R}$, gives an unique signature of the phonon mode.

Analogously, in our calculations, we considered the difference between the calculated DR/R curves at 0 and $\pi$, multiplied by an exponential factor derived from the experimental data, to account for the damping of the optical modulation. The DFT predictions closely match the experimental results for both the 8 and $80 \mathrm{~cm}^{-1}$ modes by rescaling the atomic displacements, whose relative amplitude is given by DFPT, with a global multiplicative factor, common to both polarizations (Fig. 4). The agreement is particularly good for $\eta \| \mathbf{x}$, where the phonon effects are most prominent. Peculiar features, such as their trend and change of sign, are maintained throughout the relaxation process as previously illustrated in Figs. 2(c) and $2(\mathrm{~d})$.

From this comparison, we can quantify the magnitude of the ionic displacements right after the perturbation, when the atoms of different unit cells move uniformly in the probed region. The estimated displacements have to be regarded as average displacements over the probed volume and photon energies. The penetration depth, derived from the optical data in Ref. [31], is almost constant and similar for the two perpendicular polarizations in the probed spectral region, with minor deviations toward the infrared for the $\mathbf{y}$ direction.

In order to evaluate very small atomic displacements, we repeated the experiments at lower fluence $\left(\approx 230 \mu \mathrm{J} / \mathrm{cm}^{2}\right)$. We checked that the DR/R spectral shape does not change 

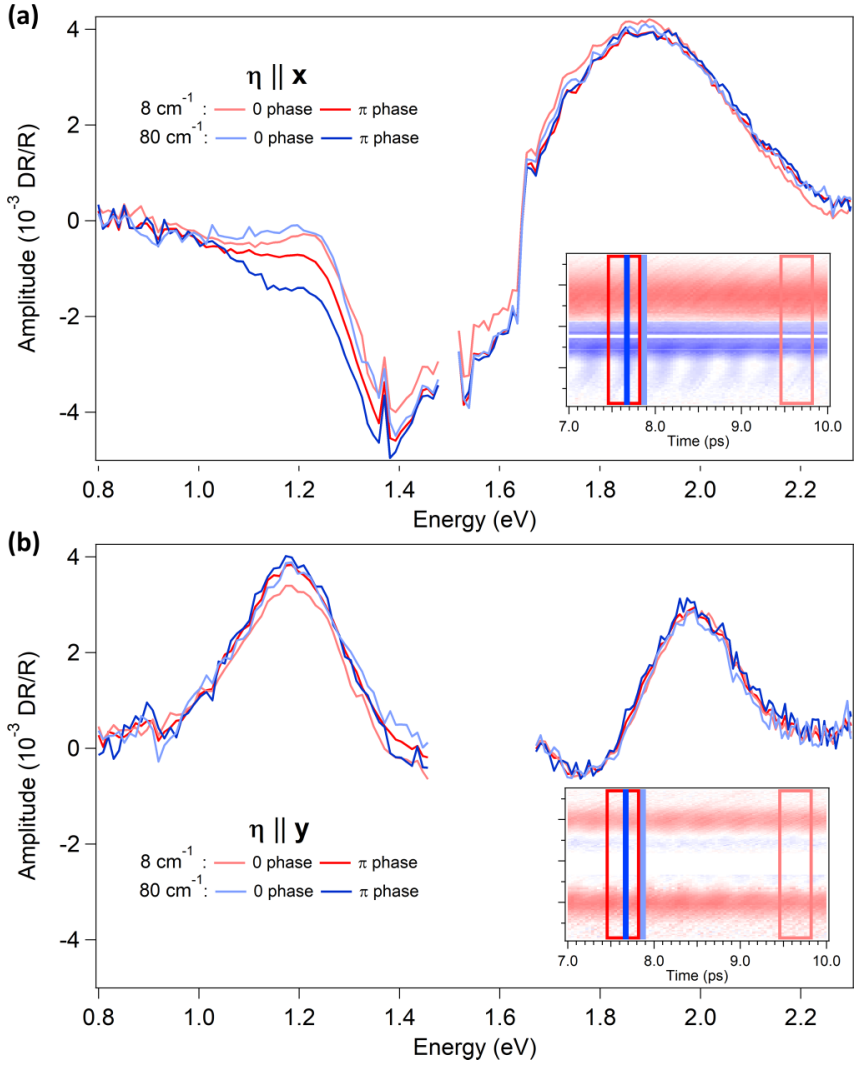

FIG. 3. Experimental spectral profiles extracted from the $\mathrm{DR} / \mathrm{R}(t, h v)$ maps reported in Figs. 1(c) and 1(d). The linearly polarized probe is oriented as (a) $\eta \| \mathbf{x}$ and (b) $\eta \| \mathbf{y}$. The 0 and $\pi$ phases are referred to the maximum and minimum of the oscillation in the DR/R signal for $\eta \| \mathbf{x}$ in the infrared spectral region; the insets show the time delays at which the curves were extracted. The vertical axis and color scale of the insets are the same as for Figs. 1(c) and $1(\mathrm{~d})$.

at lower temperature or lower fluence, where we showed that the phonon effects can be considered linear with the deposited energy [20]. This is not the case for $\approx 710 \mu \mathrm{J} / \mathrm{cm}^{2}$, since the phonon effects measured at that fluence are only twice those at $\approx 230 \mu \mathrm{J} / \mathrm{cm}^{2}$. At $\approx 230 \mu \mathrm{J} / \mathrm{cm}^{2}$, for the $8 \mathrm{~cm}^{-1}$ mode, the initial positions shifts are found to be $\approx 350 \mathrm{fm}$ along the y direction. For the $80 \mathrm{~cm}^{-1}$ mode, the displacements are quite distinct for the twelve atoms in the basis cell. The four tungsten atoms remain almost fixed (displacements smaller than $10 \mathrm{fm}$ ) along the $\mathbf{y}$ direction whereas they shift by $\approx 40 \mathrm{fm}$ along the interlayer $\mathbf{z}$ direction. Four tellurium atoms are displaced by $\approx 90 \mathrm{fm}$ along the $\mathbf{y}$ direction and by $\approx 25 \mathrm{fm}$ along $\mathbf{z}$. The remaining four tellurium atoms are displaced by $\approx 15 \mathrm{fm}$ along the $\mathbf{y}$ direction and by $\approx 30 \mathrm{fm}$ along $\mathbf{z}$. None of these modes involves movements along the $\mathbf{x}$ crystallographic direction.

We highlight that with the present approach the atomic displacements can be evaluated with a precision of a few femtometers without free tuning parameters, except a scaling factor determined by an overall comparison between experimental data and numerical simulations of the broadband $\mathrm{DR} / \mathrm{R}$ signal. Concerning $\mathrm{WTe}_{2}$, a good agreement between the measured and calculated phonon effects on the optical
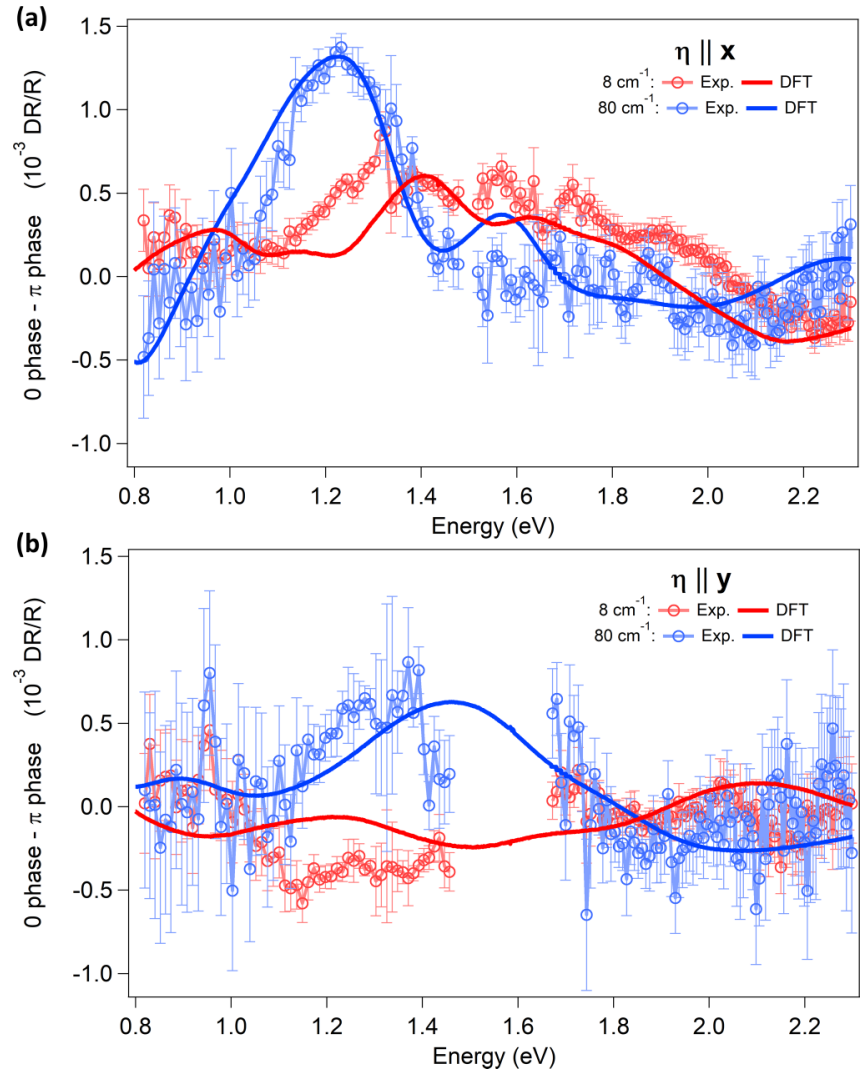

FIG. 4. Comparisons between the experimental and DFT calculated DR/R difference between the 0 and $\pi$ phases, showcasing the effects of the 8 and $80 \mathrm{~cm}^{-1}$ optical phonons for (a) $\eta \| \mathbf{x}$ and (b) $\eta \| \mathbf{y}$ using an $\approx 710 \mu \mathrm{J} / \mathrm{cm}^{2}$ absorbed pump fluence and $T=295 \mathrm{~K}$.

properties for both in-plane crystal axes has been obtained. This finding confirms that the macroscopic lattice distortions excited in $\mathrm{WTe}_{2}$ at 8 and $80 \mathrm{~cm}^{-1}$ can be entirely mimicked by the coherent population of selected zone-center $\mathrm{A}_{1}$ lattice modes. We stress that the possibility of measuring the optical properties on a wide spectral range is fundamental to extracting reliable quantitative results about the magnitude of eigendisplacements of multiple and intertwined phonon modes. Finally, we mention that the method described here is not system specific and in principle can be extended to any crystalline material, provided that its high-energy optical properties are affected by the coherent motion of atoms. Our findings can pave the way to the design of tailored devices in which the coherent lattice motion (at selected frequencies and up to large displacements) is exploited to finely tune the functional properties of semiconducting and metallic systems.

We acknowledge fruitful discussion with and suggestions concerning the use of the YAMBO code from Davide Sangalli and Andrea Marini. We thank Paolo Giannozzi for useful discussions. D.S. acknowledges support from the European Social Fund Operational Programme 2014/2020 of Region Friuli Venezia Giulia. High-performance computing resources were obtained from CINECA through the ISCRA initiative and the agreement with the University of Trieste and from Elettra Sincrotrone Trieste. R.J.C. acknowledges that 
funding for the growth of the crystals used in this work was provided by the US NSF MRSEC program, Grant No. DMR-1420541. F.P. and M.P. acknowledge the University of
Trieste for supporting this research with the Projects FRA 2016, Code No. J96J16000980005, and FRA 2018, Code No. J91G18000730002, respectively.
[1] N. Ashcroft and N. Mermin, Solid State Physics (Saunders College, Philadelphia, 1976), Chap. 21.

[2] M. Rini, R. Tobey, N. Dean, J. Itatani, Y. Tomioka, Y. Tokura, R. W. Schoenlein, and A. Cavalleri, Nature (London) 449, 72 (2007).

[3] D. Nicoletti and A. Cavalleri, Adv. Opt. Photon. 8, 401 (2016).

[4] T. Dekorsy, G. C. Cho, and H. Kurz, in Light Scattering in Solids VIII: Fullerenes, Semiconductor Surfaces, Coherent Phonons (Springer, Berlin, 2000), pp. 169-209.

[5] K. Ishioka and O. V. Misochko, in Progress in Ultrafast Intense Laser Science (Springer, Berlin, 2010), Vol. 5, pp. 23-46.

[6] A. V. Kuznetsov and C. J. Stanton, in Hot Carriers in Semiconductors (Springer US, Boston, MA, 1996), pp. 217-220.

[7] K. Ishioka, M. Hase, M. Kitajima, and H. Petek, Appl. Phys. Lett. 89, 231916 (2006).

[8] A. Melnikov, O. Misochko, and S. Chekalin, Phys. Lett. A 375, 2017 (2011).

[9] S.-H. Kim, B.-J. Kim, T.-Y. Jeong, Y.-S. Lee, and K.-J. Yee, J. Appl. Phys. 117, 163107 (2015).

[10] K. Ishioka, K. Brixius, A. Beyer, A. Rustagi, C. J. Stanton, W. Stolz, K. Volz, U. Höfer, and H. Petek, Appl. Phys. Lett. 108, 051607 (2016).

[11] K. Sokolowski-Tinten, C. Blome, J. Blums, A. Cavalleri, C. Dietrich, A. Tarasevitch, I. Uschmann, E. Förster, M. Kammler, M. Horn-von Hoegen et al., Nature (London) 422, 287 (2003).

[12] R. P. Chatelain, V. R. Morrison, B. L. M. Klarenaar, and B. J. Siwick, Phys. Rev. Lett. 113, 235502 (2014).

[13] T. K. Cheng, S. D. Brorson, A. S. Kazeroonian, J. S. Moodera, G. Dresselhaus, M. S. Dresselhaus, and E. P. Ippen, Appl. Phys. Lett. 57, 1004 (1990).

[14] Y. Kaoru, G. Antonio, and L. Kenneth, Progress in Ultrafast Intense Laser Science (Springer, Berlin, 2010), Vol. 5, Chap. 2.

[15] M. F. DeCamp, D. A. Reis, P. H. Bucksbaum, and R. Merlin, Phys. Rev. B 64, 092301 (2001).

[16] H. Katsuki, J. C. Delagnes, K. Hosaka, K. Ishioka, H. Chiba, E. S. Zijlstra, M. E. Garcia, H. Takahashi, K. Watanabe, M. Kitajima et al., Nat. Commun. 4, 2801 (2013).

[17] M. N. Ali, J. Xiong, S. Flynn, J. Tao, Q. D. Gibson, L. M. Schoop, T. Liang, N. Haldolaarachchige, M. Hirschberger, N. P. Ong et al., Nature (London) 514, 205 (2014).
[18] A. A. Soluyanov, D. Gresch, Z. Wang, Q. Wu, M. Troyer, X. Dai, and B. A. Bernevig, Nature (London) 527, 495 (2015).

[19] A. Mar, S. Jobic, and J. A. Ibers, J. Am. Chem. Soc. 114, 8963 (1992).

[20] See Supplemental Material at http://link.aps.org/supplemental/ 10.1103/PhysRevResearch.1.032033 for complementary experimental and computational details.

[21] B. Amin, T. P. Kaloni, and U. Schwingenschlögl, RSC Adv. 4 34561 (2014).

[22] E. J. Sie, C. M. Nyby, C. D. Pemmaraju, S. J. Park, X. Shen, J. Yang, M. C. Hoffmann, B. K. Ofori-Okai, R. Li, A. H. Reid et al., Nature (London) 565, 61 (2019).

[23] P. K. Das, D. Di Sante, I. Vobornik, J. Fujii, T. Okuda, E. Bruyer, A. Gyenis, B. E. Feldman, J. Tao, R. Ciancio et al., Nat. Commun. 7, 10847 (2016).

[24] D. R. Hamann, M. Schlüter, and C. Chiang, Phys. Rev. Lett. 43, 1494 (1979).

[25] T. Takeda, Z. Phys. B 32, 43 (1978).

[26] J. P. Perdew, J. A. Chevary, S. H. Vosko, K. A. Jackson, M. R. Pederson, D. J. Singh, and C. Fiolhais, Phys. Rev. B 46, 6671 (1992).

[27] D. R. Hamann, Phys. Rev. B 88, 085117 (2013).

[28] M. van Setten, M. Giantomassi, E. Bousquet, M. Verstraete, D. Hamann, X. Gonze, and G.-M. Rignanese, Comput. Phys. Commun. 226, 39 (2018).

[29] P. Giannozzi, S. Baroni, N. Bonini, M. Calandra, R. Car, C. Cavazzoni, D. Ceresoli, G. L. Chiarotti, M. Cococcioni, I. Dabo et al., J. Phys.: Condens. Matter 21, 395502 (2009).

[30] A. Marini, C. Hogan, M. Grüning, and D. Varsano, Comput. Phys. Commun. 180, 1392 (2009)

[31] A. J. Frenzel, C. C. Homes, Q. D. Gibson, Y. M. Shao, K. W. Post, A. Charnukha, R. J. Cava, and D. N. Basov, Phys. Rev. B 95, 245140 (2017).

[32] M. Ligges, I. Rajkovic, P. Zhou, O. Posth, C. Hassel, G. Dumpich, and D. von der Linde, Appl. Phys. Lett. 94, 101910 (2009).

[33] Y. M. Dai, J. Bowlan, H. Li, H. Miao, S. F. Wu, W. D. Kong, P. Richard, Y. G. Shi, S. A. Trugman, J.-X. Zhu et al., Phys. Rev. B 92, 161104(R) (2015).

[34] B. He, C. Zhang, W. Zhu, Y. Li, S. Liu, X. Zhu, X. Wu, X. Wang, H. Wen, and M. Xiao, Sci. Rep. 6, 30487 (2016). 